BMJ Open

Diabetes

Research

\& Care

\section{An observational study of patient characteristics and mortality following hypoglycemia in the community}

To cite: Elwen FR, Huskinson A, Clapham L, et al. An observational study of patient characteristics and mortality following hypoglycemia in the community. BMJ Open Diabetes Research and Care 2015;3: 000094.

doi:10.1136/bmjdrc-2015000094

FRE and $\mathrm{AH}$ are joint first authors.

Received 19 February 2015 Revised 18 May 2015 Accepted 22 May 2015

\section{(a) CrossMark}

${ }^{1}$ Division of Cardiovascular and Diabetes Research, Leeds Institute of Cardiovascular and Metabolic Medicine (LICAMM), The University of Leeds, Leeds, UK

${ }^{2}$ The Leeds Teaching Hospitals Trust, St James' University Hospital, Leeds, UK

${ }^{3}$ Academic Unit of Diabetes, Endocrinology and Metabolism, University of Sheffield, Sheffield, UK ${ }^{4}$ Yorkshire Ambulance Services, Wakefield, UK

Correspondence to Dr Ramzi A Ajjan; r.ajjan@leeds.ac.uk

\section{ABSTRACT}

Objectives: Characterize patients with diabetes with severe hypoglycemia requiring emergency services intervention at home and investigate 12-month mortality.

Research design and methods: Emergency services call-outs for hypoglycemia were recorded between 2005 and 2013 in an area covering 34000 patients with diabetes. Patient characteristics were documented together with capillary blood glucose (CBG), glycated hemoglobin (HbA1c), and treatment for hypoglycemia; 12-month mortality and variables influencing survival were analyzed.

Results: In 1835 episodes among 1156 patients, 45\% had type 1 diabetes (68.2\% males) and $44 \%$ had type 2 diabetes (49.4\% males), with a minority unclassified. $\mathrm{CBG}$ at presentation $\left(\mathrm{mean}_{ \pm} \mathrm{SD}\right.$ ) was $1.76 \pm 0.72 \mathrm{mmol} / \mathrm{L}$ in patients with type 1 diabetes and $1.96 \pm 0.68 \mathrm{mmol} / \mathrm{L}$ in patients with type 2 diabetes $(p<0.0001)$, with a higher $\mathrm{HbA} 1 \mathrm{C}$ in the former group $(8.3 \pm 1.52 \%$ (67.5 $\pm 16.4 \mathrm{mmol} / \mathrm{mol})$ and $7.8 \pm 1.74 \%(61.6 \pm 19.0 \mathrm{mmol} /$ $\mathrm{mol}$ ), respectively; $p<0.0001)$. A third of patients with type 2 diabetes were not on insulin therapy and displayed lower HbA1c compared with insulin users. Glucagon was used in $37 \%$ of patients with type 1 diabetes and $28 \%$ of patients with type 2 diabetes $(p<0.0001)$. One-year mortality was $4.45 \%$ in type 1 diabetes and $22.1 \%$ in type 2 diabetes. Age and type of diabetes were predictive of mortality in multivariable analysis, whereas CBG levels/frequency of hypoglycemia had no effect.

Conclusions: Severe hypoglycemia in the community is common with a male predominance in type 1 diabetes. Severe hypoglycemia in non-insulin treated patients with type 2 diabetes is associated with lower HbA1c compared with insulin users. Severe hypoglycemia appears to be associated with increased mortality at 12 months, particularly in type 2 diabetes.

\section{INTRODUCTION}

Hypoglycemia is a common side effect of glucose-lowering treatment in diabetes mellitus (DM) and may have serious clinical consequences in the short and long terms. ${ }^{1}$ Many episodes of hypoglycemia, even severe, are managed at home by the patient or carer

\section{Key messages}

- Severe hypoglycemia in the community is common, and presents a large burden on both patients and healthcare workers.

- Using a large database of ambulance call-outs for hypoglycemia, this study aimed to characterize those requiring the emergency services for an episode of hypoglycemia, and to investigate factors that may be associated with an increased risk of mortality.

- We found that a third of patients with type 2 diabetes having severe hypoglycemic episodes were not using any insulin; these individuals had a lower glycated hemoglobin than those with type 2 diabetes requiring insulin treatment.

- Twelve month mortality following an episode of severe hypoglycemia was high, especially in individuals with type 2 diabetes. More research is required to investigate the cause of death in these patients.

and rarely require intervention of the ambulance services or admission to hospital.

Severe hypoglycemia in diabetes, defined as an episode requiring external assistance, causes a significant burden on patients and medical staff. Although most episodes of hypoglycemia are managed by the patient or family members/carers, studies estimate incidence rates of 11.5 and 11.8 per 100 patient years that require emergency medical service intervention in type 1 diabetes and insulintreated type 2 diabetes, respectively. ${ }^{2}$ Severe hypoglycemia was previously thought to be predominantly related to insulin therapy, but more recent work indicates that this occurs with oral hypoglycemia agents (OHAs), largely involving agents in the sulfonylurea group. $^{2-4} \mathrm{~A}$ recent meta-analysis has shown that almost a fifth of patients on sulfonylurea suffer at least one hypoglycemic episode, with the risk of low blood glucose increasing more than threefold with the use of these agents. ${ }^{5}$ Although the incidence of severe hypoglycemia is relatively low at $1.2 \%$, the 
large number of patients treated with sulfonylurea translates clinically into a significant number of individuals suffering this serious complication.

Tight control of the blood glucose level in DM reduces the risk of developing microvascular complications, but the effect on macrovascular disease is less clear, at least in the medium term. ${ }^{6-12}$ Intensive glycemic treatment carries an increased incidence of hypoglycemia, which is associated with increased mortality, including cardiovascular death. ${ }^{8}{ }^{13-18}$ Two large trials investigating the role of intensive treatment in patients with type 2 diabetes demonstrated that having one or more episodes of severe hypoglycemia is associated with increased mortality. ${ }^{8} 918$ Recent retrospective studies have also found an increased mortality rate associated with hypoglycemia in diabetes, in hospital and community settings. ${ }^{19-21}$ It should be stressed that the association between hypoglycemia and mortality does not necessarily indicate causation. Low blood glucose may be a marker of morbidity and frailty; however, the results of a large recent meta-analysis suggest that this would not entirely explain the association. ${ }^{22}$

Although large studies attempt to reduce bias and risk of confounding, the randomized nature of a number of such studies and the selected population investigated are major caveats, making general applicability of the results uncertain. Therefore, more work is needed to clarify the relationship between hypoglycemia and mortality in the diabetes population in real-life situations. In particular, the characteristics of patients prone to severe hypoglycemia are not entirely clear and factors that may contribute to mortality following hypoglycemia require further analysis.

The current study therefore aimed to: (1) characterize a large cohort of patients with diabetes with severe hypoglycemia requiring emergency services intervention while at home, (2) investigate mortality at 12 months following severe hypoglycemia and analyze the contributing factors to identify the subpopulation at risk.

\section{METHODS}

\section{Study population and collection of basic data}

Data have been collected on an emergency services call-out for hypoglycemia between July 2005 and April 2013 by the project coordinator among a population of approximately 34000 participants with diabetes. Individuals were included in the analysis if capillary blood glucose (CBG) levels were less than $4 \mathrm{mmol} / \mathrm{L}$ at attendance of the emergency crew. Basic characteristics were collected by the Yorkshire Ambulance Services (YAS) at the time of the episode, including basic patient demographics (age, gender and type of diabetes). Diabetes treatment was also recorded including prescribed OHA and/or insulin therapy. CBG was measured at the arrival of ambulance services and following treatment of hypoglycemia. Low blood glucose in these individuals was treated by food/drink, glucagon, and/or intravenous dextrose. Data on whether the individual required transport to hospital were also collected. In a minority of patients when the type of diabetes was unknown, patients less than 30 years and using insulin were assumed to have type 1 diabetes and patients using one or more oral OHA(s) were assumed to have type 2 diabetes. All data were thoroughly examined for individuals with multiple episodes, which were clarified from the database. Patients gave consent for referral into the pathway which aimed for a diabetes specialist nurse to contact the patient by phone within 5 days of the hypoglycemic episode to try to establish a cause and offer advice to prevent further episodes.

The latest glycated hemoglobin (HbAlc) data before the incident were recorded by the diabetes nurse specialist as part of the hypoglycemia pathway before anonymized patient data, including pretreatment CBG and treatment received, were passed to the research team. Permission to undertake the study was given by the local ethical authority.

\section{Mortality data}

A randomly selected 3-year sample was taken for mortality analysis (episodes between April 2009 and April 2012) after no statistically significant differences were found between the 3-year sample and the whole data set in regard to the gender distribution, the type of diabetes, mean age, mean pretreatment blood glucose and whether patients were transported to the hospital. Data concerning 1 year mortality following the last recorded hypoglycemic episode were collected using a coding system in the local database and date of death was recorded. We were unable to locate the mortality status of 11 patients; they were therefore excluded from the analysis.

\section{Procedures and statistical analyses}

We used SPSS (V.22.0) for statistical analyses. Normally distributed data are displayed as means and SDs, unless otherwise stated, and were analyzed using a two-tailed $\mathrm{t}$ test. Non-normally distributed data are displayed as median and IQR, with the Mann-Whitney $\mathrm{U}$ test used to assess differences. Categorical data were analyzed using the $\chi^{2}$ test. Binary logistic regression was used to analyze variables influencing 12-month mortality. In all tests, a $\mathrm{p}$ value of less than 0.05 was considered to be significant.

Multivariable analysis was used to analyze variables influencing 12-month mortality. Variables included in the model were: age, type of DM, whether they were taken to hospital after initial treatment at the scene, the number of episodes each patient had during the study period, and their pretreatment CBG on their last recorded episode. Logistic regression analysis was used to identify predictors of 12-month mortality, and Cox proportional regression models to perform survival analysis. For patients who were alive after 1 year from their hypoglycemia, a 12-month census date was used, 
whereas for patients who were deceased, the date of death was used.

\section{RESULTS}

\section{Patient characteristics}

A total of 1911 episodes were recorded by the project coordinator in 1195 patients, of which 76 were excluded for the following reasons: multiple counting $(n=14)$, gestational diabetes $(n=1)$, no history of diabetes $(n=3)$ and baseline CBG $\geq 4 \mathrm{mmol} / \mathrm{L} \quad(\mathrm{n}=58)$, as summarized in figure 1 . The remaining 1835 episodes occurred among 1156 patients, of which $520(45 \%)$ had type 1 diabetes and $503(44 \%)$ had type 2 diabetes, with the rest of the patients unclassified (11\%). Of these individuals, 880 (76\%) patients had a single hypoglycemic event compared with 276 (24\%) who had multiple hypoglycemic episodes during the study period. The main characteristics of patients are summarized in table 1 .

Overall, a pretreatment CBG of less than $3.0 \mathrm{mmol} / \mathrm{L}$ was found in $1643(90 \%)$ episodes with approximately $80 \%$ of episodes recording a CBG of less than $2.5 \mathrm{mmol} / \mathrm{L}$.

\section{Blood glucose levels and HbA1c}

The mean $( \pm \mathrm{SD})$ pretreatment $\mathrm{CBG}$ in individuals with type 1 diabetes was significantly lower than that in individuals with type 2 diabetes $(1.76 \pm 0.72$ vs 1.96 $\pm 0.68 \mathrm{mmol} / \mathrm{L}$, respectively; $\mathrm{p}<0.0001)$. The reverse was true for HbA1c with a mean of $8.3 \pm 1.52 \% \quad(67.5$ $\pm 16.4 \mathrm{mmol} / \mathrm{mol}$ ) in type 1 diabetes compared with 7.8 $\pm 1.74 \% \quad(61.6 \pm 19.0 \mathrm{mmol} / \mathrm{mol})$ for type 2 diabetes $(\mathrm{p}<0.0001)$. When comparing HbAlc between genders in type 1 diabetes, a mean of $8.3 \pm 1.41 \% \quad(67.4$ $\pm 15.1 \mathrm{mmol} / \mathrm{mol}$ ) was found in males, which was similar to females at $8.3 \pm 1.82 \%(67.7 \pm 19.3 \mathrm{mmol} / \mathrm{mol} ; \mathrm{p}=0.86)$.

\section{Treatment for hypoglycemia}

This included: food and drink (including the use of glucose gel), intravenous glucose, intramuscular glucagon or none. Episodes in which food and drink were

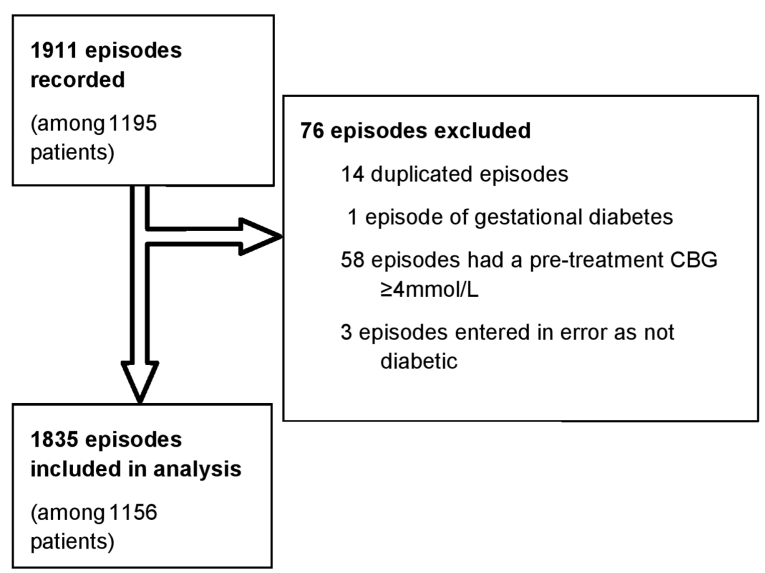

Figure 1 Flow diagram showing episodes excluded from data analysis. $\mathrm{CBG}=$ capillary blood glucose. given had a significantly $(\mathrm{p}<0.0001)$ higher mean pretreatment CBG $(2.21 \pm 0.73 \mathrm{mmol} / \mathrm{L})$ compared to those in which glucagon $(1.61 \pm 0.60 \mathrm{mmol} / \mathrm{L})$ or intravenous glucose $(1.67 \pm 0.63 \mathrm{mmol} / \mathrm{L})$ were used. Details of hypoglycemic treatment are provided in table 1 . There was marginally higher glucose levels post-treatment in patients with type 1 diabetes compared with those with type 2 diabetes $(6.41 \pm 2.71$ vs $6.15 \pm 2.59 \mathrm{mmol} / \mathrm{L}$, respectively; $\mathrm{p}=0.049$ ). There was no difference in the type of treatment administered for hypoglycemia when comparing patients with type 2 diabetes on insulin with those using OHAs alone.

\section{Transport to hospital}

Of all episodes, 123 (7\%) were subsequently transported to hospital. Those transported were older (median age 71 years $(51-80)$ vs $60(43-74), \mathrm{p}=0.0017)$, more likely to have type 2 diabetes than type 1 diabetes (9\% vs $4 \%$, respectively; $\mathrm{p}=0.0001)$, lived alone $(37 \%$ vs $24 \%$, respectively; $\mathrm{p}=0.0012$ ), and were more likely to have had just one episode compared to multiple previous episodes $(67 \%$ vs $33 \%$, respectively; $\mathrm{p}<0.0001)$.

\section{Insulin and non-insulin-treated patients with type 2 diabetes}

Two-thirds of individuals with type 2 diabetes (69\%) were on insulin treatment, and this group included those having insulin alone or in combination with OHA(s). Among $2 \%$ of episodes, the treatment type remained unknown, but the remaining $29 \%$ were on OHA alone. Within the subset using solely OHAs, 159 episodes (82\%) involved a sulfonylurea either independently or in conjunction with one or more OHA. Only $3 \%$ of episodes occurred with OHA not usually associated with hypoglycemia, including metformin. In 30 of the remaining episodes $(15 \%)$, the type of OHA used was unknown, and therefore sulfonylurea use may have been even higher. Individuals with type 2 diabetes on OHA treatment alone had a significantly lower HbA1c

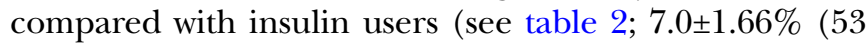
$\pm 18.0 \mathrm{mmol} / \mathrm{mol})$ vs $8.3 \pm 1.56 \% \quad(67 \pm 17.0 \mathrm{mmol} / \mathrm{mol})$, respectively; $\mathrm{p}<0.0001$.

\section{Outcome 1 year following hypoglycemia}

Given the uniformity of data, we conducted 12-month mortality analysis in a 3-year sample taken between April 2009 and April 2012 (739 episodes in 510 patients). The characteristics of these patients were similar to those of the study group as a whole (data not shown). Of these 510 patients, 67 died within a year, giving a 12-month mortality rate of $13.1 \%$. The median age of patients who died within a year of their last recorded episode was 77 years (72-82). There were 10 patients who had died within a year who had an unknown type of diabetes.

There were a total of 11 patients ( 6 male, 5 female) with type 1 diabetes who were found to have died 1 year after their last presentation, giving a 12-month mortality rate of $4.45 \%$ with a median age of $54(43-67)$ years. 
Table 1 Basic characteristics of all hypoglycemic episodes as well as those among individuals with T1DM and T2DM

\begin{tabular}{|c|c|c|c|c|}
\hline & $\begin{array}{l}\text { All episodes } \\
(n=1835)\end{array}$ & $\begin{array}{l}\text { Patients with T1DM } \\
(n=1015)\end{array}$ & $\begin{array}{l}\text { Patients with T2DM } \\
(n=664)\end{array}$ & p Value \\
\hline Median age (years) & $61(44-75)$ & $48(35-61)$ & $75(68-81)$ & $<0.0001$ \\
\hline Females (\%) & $732(40 \%)$ & $323(32 \%)$ & $336(51 \%)$ & $<0.0001$ \\
\hline Pretreatment CBG (mmol/L) & $1.85( \pm 0.72)$ & $1.76( \pm 0.72)$ & $1.96( \pm 0.68)$ & $<0.0001$ \\
\hline Post-treatment CBG (mmol/L) & $6.30( \pm 2.66)$ & $6.41( \pm 2.71)$ & $6.15( \pm 2.59)$ & 0.0494 \\
\hline Mean $\mathrm{HbA} 1 \mathrm{c}(\mathrm{mmol} / \mathrm{mol})$ & $65.0( \pm 17.8)$ & $67.5( \pm 16.4)$ & $61.6( \pm 19.0)$ & $<0.0001$ \\
\hline Insulin use & $1600(87 \%)$ & $1015(100 \%)$ & $448(69 \%)$ & \\
\hline \multicolumn{5}{|l|}{ Treatment given } \\
\hline Food and drink & $640(35 \%)$ & $314(31 \%)$ & $272(41 \%)$ & $<0.001$ \\
\hline IV Glucose & $531(29 \%)$ & $294(29 \%)$ & $187(28 \%)$ & 0.722 \\
\hline Glucagon & 609 (33\%) & $379(37 \%)$ & $187(28 \%)$ & $<0.001$ \\
\hline None & $22(1 \%)$ & $14(1 \%)$ & $6(1 \%)$ & 0.38 \\
\hline Transported to hospital & $123(7 \%)$ & $40(4 \%)$ & $59(9 \%)$ & 0.0018 \\
\hline Lives alone & $463(25 \%)$ & $221(21 \%)$ & $199(30 \%)$ & $<0.0001$ \\
\hline
\end{tabular}

Data are presented as absolute numbers (percentages), median (IQR) or mean (SD).

CBG, capillary blood glucose; HbA1c, glycated hemoglobin; IV, intravenous; T1DM, type 1 diabetes; T2DM, type 2 diabetes.

There were 46 patients (23 male, 23 female) with type 2 diabetes who had died within a year, giving a 12-month mortality rate of $22.1 \%$ with a median age of 78 (74-82) years. There was no significant difference in mortality rates as to whether patients with type 2 diabetes were on insulin or non-insulin therapy for the management of glycemia (data not shown). Of the 67 patients who died 12 months after their last episode, $38.8 \%(n=26)$ of these deaths occurred in the first 3 months (giving a 3-month mortality rate of $5.1 \%$ ). Excluding patients with type 2 diabetes with an age $\geq 75$ years, which gave a median age of 67 years $(60-72)$, 12 -month mortality fell to $14.3 \%$. The mortality rate of those patients with type 2 diabetes aged $\geq 75$ years was $27.4 \%$ (median age 81 years, IQR 78-84).

\section{Predictors of mortality}

A model was used to find significant predictive variables for 12-month mortality, which included age, type of diabetes, transport to hospital after the episode, pretreatment blood glucose and multiple episodes within the past 3 years.
In univariate analysis, age and type of diabetes were predictors of mortality with ORs of 1.056 (1.036 to $1.077)$ and 6.092 (3.063 to 12.116), respectively. Transport to hospital only showed a trend, whereas severity of hypoglycemia or number of episodes showed no predictive value (see table 3). Multivariate analysis yielded similar data, with the effect of age and type of diabetes on mortality not confounded by other variables.

\section{Survival analysis}

Survival analysis was completed using a Cox proportional hazards model to further investigate the effect of different variables on mortality. Results of survival analysis were consistent with logistic regression, with age (HR 1.025 (1.006 to 1.045$), \mathrm{p}=0.011$ ) and having type 2 diabetes (HR 2.034 (1.016 to 4.072), $\mathrm{p}=0.045$ ) remaining significantly associated with an increased risk of death.

\section{CONCLUSIONS}

This is the largest study to investigate, over a period of 8 years, the main characteristics of patients with diabetes

Table 2 Characteristics of episodes among those with type 2 diabetes by treatment modality

\begin{tabular}{|c|c|c|c|c|}
\hline & Insulin with or without OHA & OHA alone & Not known & p Value \\
\hline Episodes & $458(69 \%)$ & $195(29 \%)$ & $11(2 \%)$ & $<0.001$ \\
\hline Pretreatment CBG (mmol/L) & $1.91( \pm 0.70)$ & $2.06( \pm 0.64)$ & & 0.0113 \\
\hline Post-treatment CBG (mmol/L) & $6.22( \pm 2.63)$ & $6.00( \pm 2.56)$ & & 0.3220 \\
\hline $\mathrm{OHA}$ use & $48(10.5 \%)$ & & & \\
\hline Sulfonylurea (alone and in combo) & $14(3 \%)$ & $159(82 \%)$ & & \\
\hline Metformin alone & $26(6 \%)$ & $5(2.5 \%)$ & & \\
\hline Pioglitazone alone & 0 & $1(0.5 \%)$ & & \\
\hline Unknown & $5(1 \%)$ & $30(15 \%)$ & & \\
\hline Other & $3(0.5 \%)$ & 0 & & \\
\hline Mean $\mathrm{HbA} 1 \mathrm{c}(\mathrm{mmol} / \mathrm{mol})$ & $67.0( \pm 17.0)$ & $53.0( \pm 18.0)$ & & $<0.0001$ \\
\hline
\end{tabular}

CBG, capillary blood glucose, $\mathrm{HbA1c}$, glycated hemoglobin; OHA, oral hypoglycemic agent. 
Table 3 Univariate and multivariate analysis of factors predicting mortality 12 months after the last hypoglycemic episode recorded between April 2009 and April 2012

\begin{tabular}{|c|c|c|c|c|}
\hline & \multicolumn{2}{|l|}{ Univariate } & \multicolumn{2}{|l|}{ Multivariate } \\
\hline & OR (95\% Cl) & p Value & OR (95\% Cl) & p Value \\
\hline Age & 1.056 (1.036 to 1.077$)$ & $<0.001$ & 1.041 (1.014 to 1.069$)$ & 0.002 \\
\hline Type of diabetes (type 2 diabetes) & 6.092 (3.063 to 12.116$)$ & $<0.001$ & $2.468(1.025$ to 5.944$)$ & 0.044 \\
\hline Transported after episode & $1.935(0.940$ to 3.984$)$ & 0.073 & $2.276(0.995$ to 5.207$)$ & 0.052 \\
\hline Pretreatment CBG & 0.961 (0679 to 1.362$)$ & 0.824 & 0.691 (0.440 to 1.086$)$ & 0.109 \\
\hline$>1$ episode & 1.161 (0.619 to 2.178$)$ & 0.641 & $0.747(0.352$ to 1.584$)$ & 0.447 \\
\hline
\end{tabular}

who develop severe hypoglycemia in the community requiring emergency services intervention. Moreover, this work analyzed mortality within 12 months of the hypoglycemic episode, to further clarify the relationship between low blood glucose and medium-term clinical outcome.

Our study showed: (1) Patients with diabetes requiring emergency services intervention for hypoglycemia were approximately equally divided between patients with type 1 diabetes and type 2 diabetes with more severe hypoglycemia documented in the former group, (2) gender distribution was similar in those with type 2 diabetes, but a male predominance was evident in type 1 diabetes, (3) treatment to reverse hypoglycemia was different comparing type 1 diabetes with type 2 diabetes, with a more frequent use of glucagon in the former group, (4) a third of patients with type 2 diabetes were not on insulin treatment and had a lower HbAlc compared with the insulin-treated group, (5) mortality was high within 12 months of the hypoglycemic event, particularly in participants with type 2 diabetes.

The severity of hypoglycemia requiring emergency services intervention appeared more profound in individuals with type 1 diabetes compared with those with type 2 diabetes. This may be due to the former group being more able to treat mild hypoglycemia at home without emergency services input, or it may relate to impaired defences against hypoglycemia in those with type 1 diabetes. In support of the former theory, a study in 2005 demonstrated that among people with type 1 diabetes, the majority of episodes of severe hypoglycaemia were treated by friends or relatives and did not require emergency service intervention. ${ }^{23}$ Hence, those episodes in which emergency service help was sought are presumably the most severe as the individuals' normal treatment measures at home had failed. The more severe hypoglycemia in type 1 diabetes led to different treatment strategies to reverse low blood sugar, including the use of intravenous glucose and glucagon. This, in turn, may explain the higher post-treatment glucose levels in this group. Another explanation is related to the treatment administered, as OHAs have a more sustained glucose-lowering effect compared with insulin. However, the failure to detect a difference in post-treatment glucose levels in insulin and non-insulin users within the type 2 diabetes group argues against this explanation. The bias toward a male gender distribution in patients with type 1 diabetes was a consistent and potentially interesting finding. Although we are unable to provide a clear explanation for this observation, one possibility would have been related to gender differences in glycemic control. However, this was ruled out by demonstrating similar HbAlc in males and females with type 1 diabetes. An alternative explanation may be related to the recently documented link between 'fear of hypoglycemia' and female gender. ${ }^{24} 25$ It is possible that the enhanced fear of hypoglycemia in females with type 1 diabetes results in more frequent glucose testing, consequently 'protecting' from severe hypoglycemia that requires emergency services intervention. Future work is required to systematically analyze the reasons for this apparent gender difference.

In patients with type 2 diabetes, glucose levels in insulin and non-insulin-treated participants at presentation were not that dissimilar, indicating that OHA can induce severe hypoglycemia to a similar degree as insulin. Not unexpectedly, the majority of OHA-treated patients were on sulfonylurea therapy, which is an established second-line therapy in diabetes due to the known side effect profile and low cost. It should be noted that no difference in treatment to reverse hypoglycemia was observed in insulin and non-insulin-treated patients and the rate of hospital transfer was similar in the two groups. However, a clear difference was detected in $\mathrm{HbA1c}$ with OHA-treated patients displaying significantly lower levels of HbA1c compared with insulin users.

A major finding of this study was the high rate of mortality within a year of the hypoglycemic event, with a 12-month mortality rate of $13.1 \%$ and a 3 -month mortality rate of $5.1 \%$. Although the observational nature of data cannot provide evidence for causal relationship, the close association between hypoglycemia and mortality requires future investigation, as recent meta-analysis has shown that confounding factors (such as severe illness) may not explain the association alone. ${ }^{22}$ Mortality was particularly pronounced in individuals with type 2 diabetes and was similar to mortality following myocardial infarction in a similar group of patients. ${ }^{26}$ Our data are consistent with a recent study showing as association between hypoglycemia and mortality in insulin-treated patients with diabetes who 
were older than 30 years. $^{27}$ In contrast to our study, however, the authors failed to assess mortality in those who were not on insulin treatment.

The work has a number of strengths including the real-life clinical setting, the relatively large cohort studied, relatively good characterization of patients, and the clear distinction between patients with type 1 diabetes and those with type 2 diabetes. This is the first study to detail the treatment given and to demonstrate differences in management of patients with type 1 diabetes and type 2 diabetes following severe hypoglycemia and also differences in mortality.

However, there are a number of limitations to this study that need to be acknowledged. First, some ambulance call-outs may not have been captured, as only patients who have been recorded are analysed and therefore our data may underestimate the size of the problem. Second, some data were missing in a minority of patients, a known disadvantage of such observational studies. Third, the causes of death and various comorbidities in these patients have not been documented in this work. For example, information regarding comorbidities would help clarify the potential role of hypoglycemia in mortality or may simply indicate that low glucose levels are a marker of early demise in patients with diabetes. Finally, there are known limitations with the accuracy of CBG measurements as the patients may have been hypoglycemic for different periods of time before calling for an ambulance, and the CBG meters can also be inaccurate, especially when recording very low glucose readings. The use of the CBG values does, however, confirm that all the episodes included were genuine hypoglycemic events.

Given these limitations, a longitudinal prospective study is required to further address these deficiencies, which is currently ongoing.

In summary, our study shows that hypoglycemia in diabetes requiring emergency services intervention is common with differences demonstrated between type 1 diabetes and type 2 diabetes in relation to gender distribution, severity of hypoglycemia, type of treatment administered, and postintervention glucose levels. A third of patients with type 2 diabetes were not on insulin therapy, being mainly treated with a sulfonylurea, and some of these individuals had an inappropriately low HbA1c. Mortality rate in patients with type 1 and type 2 diabetes was high following the hypoglycemic episode, although a causative relationship is yet to be demonstrated. Future work is needed to further investigate the cause of death in these individuals and explore various management strategies aiming to reduce the unacceptably high mortality in this group of individuals.

Acknowledgements The authors would like to thank the University of Leeds for supporting AH, FRE, and MJB. The authors also wish to extend their thanks to the NIHR and LifeScan for their continuous support.

Contributors CJ and LC collected the data and helped to revise the manuscript. AH, FRE and MJB analyzed the data and wrote the manuscript. PB helped with the statistical analyses. AA and SH helped with the manuscript revision. RAA designed the study and wrote and revised the manuscript. The corresponding author will be the guarantor for this article.

Competing interests None declared.

Ethics approval Leeds West (local research ethics committee).

Provenance and peer review Not commissioned; externally peer reviewed.

Data sharing statement FE and $\mathrm{AH}$ hold the raw data which can be provided on request.

Open Access This is an Open Access article distributed in accordance with the Creative Commons Attribution Non Commercial (CC BY-NC 4.0) license, which permits others to distribute, remix, adapt, build upon this work noncommercially, and license their derivative works on different terms, provided the original work is properly cited and the use is non-commercial. See: http:// creativecommons.org/licenses/by-nc/4.0/

\section{REFERENCES}

1. Cryer PE. The barrier of hypoglycemia in diabetes. Diabetes 2008;57:3169-76.

2. Leese GP, Wang J, Broomhall J, et al. Frequency of severe hypoglycemia requiring emergency treatment in type 1 and type 2 diabetes: a population-based study of health service resource use. Diabetes Care 2003;26:1176-80.

3. $\mathrm{Ng} \mathrm{JM}$, Mellor DD, Masson EA, et al. Sulphonyurea as a cause of severe hypoglycaemia in the community. Prim Care Diabetes 2010;4:61-3.

4. UK Hypoglycaemia Study Group. Risk of hypoglycaemia in types 1 and 2 diabetes: effects of treatment modalities and their duration. Diabetologia 2007;50:1140-7.

5. Monami M, Dicembrini I, Kundisova L, et al. A meta-analysis of the hypoglycaemic risk in randomised controlled tirals with sulphonylureas in patients with type 2 diabetes. Diabetes Obes Metab 2014;16:833-40.

6. Duckworth W, Abraira C, Moritz T, et al. Glucose control and vascular complications in veterans with type 2 diabetes. New Engl J Med 2009;360:129-39.

7. The Diabetes Control and Complications Trial Research Group. The effect of intensive treatment of diabetes on the development and progression of long-term complications in insulin-dependent diabetes mellitus. New Engl J Med 1993;329:977-86.

8. Gerstein HC, Miller ME, Byington RP, et al, Action to Control Cardiovascular Risk in Diabetes Study Group. Effects of intensive glucose lowering in type 2 diabetes. New Engl J Med 2008;358:2545-59.

9. ADVANCE collaborative groupPatel A, MacMahon S, Chalmers J, et al. Intensive blood glucose control and vascular outcomes in patients with type 2 diabetes. New Engl J Med 2008;358:2560-72.

10. UK Prospective Diabetes Study (UKPDS) Group. Intensive blood-glucose control with sulphonylureas or insulin compared with conventional treatment and risk of complications in patients with type 2 diabetes (UKPDS 33). Lancet 1998;352:837-53.

11. Weidman-Evans E, Metz SM, Evans JD. Cardiovascular risks and benefits with oral drugs for Type 2 diabetes mellitus. Expert Rev Clin Pharmacol 2014;7:225-33.

12. Konig M, Lamos EM, Stein SA, et al. An insight into the recent diabetes trials: what is the best approach to prevent macrovascular and microvascular complications? Curr Diabetes Rev 2013:9:371-81.

13. Frier BM, Schernthaner G, Heller SR. Hypoglycemia and cardiovascular risks. Diabetes Care 2011;34(Suppl 2):S132-7.

14. Reno CM, Daphna-Iken D, Chen YS, et al. Severe hypoglycemia-induced lethal cardiac arrhythmias are mediated by sympathoadrenal activation. Diabetes 2013;62:3570-81.

15. Moheet A, Seaquist ER. Hypoglycemia as a driver of cardiovascular risk in diabetes. Curr Atheroscler Rep 2013;15:351.

16. Giorgino F, Leonardini A, Laviola L. Cardiovascular disease and glycemic control in type 2 diabetes: now that the dust is settling from large clinical trials. Ann NY Acad Sci 2013;1281:36-50.

17. Hsu P-F, Sung S-H, Cheng H-M, et al. Association of clinical symptomatic hypoglycemia with cardiovascular events and total mortality in type 2 diabetes: a nationwide population-based study. Diabetes Care 2013;36:894-900.

18. Zoungas S, Patel A, Chalmers J, et al. Severe hypoglycemia and risks of vascular events and death. New Engl J Med 2010;363:1410-18. 
19. McCoy RG, Van Houten HK, Ziegenfuss JY, et al. Increased mortality of patients with diabetes reporting severe hypoglycemia. Diabetes Care 2012;35:1897-901.

20. Tan HK, Flanagan D. The impact of hypoglycaemia on patients admitted to hospital with medical emergencies. Diabetic Med 2013;30:574-80.

21. Parsaik AK, Carter RE, Myers LA, et al. Hypoglycemia requiring ambulance services in patients with type 2 diabetes is associated with increased long-term mortality. Endocr Pract 2013;19:29-35.

22. Goto $\mathrm{A}$, Arah $\mathrm{OA}$, Goto $\mathrm{M}$, et al. Severe hypoglycaemia and cardiovascular disease: systematic review and meta-analysis with bias analysis. BMJ 2013;347:f4533.

23. Donnelly LA, Morris AD, Frier BM, et al. Frequency and predictors of hypoglycaemia in type 1 and insulin-treated type 2 diabetes: a population-based study. Diabet Med 2005;22:749-55.
24. Al Hayek AA, Robert AA, Braham RB, et al. Predictive risk factors for fear of hypoglycemia and anxiety-related emotional disorders among adolescents with type 1 diabetes. Med Princ Pract 2014;24:222-30.

25. Gjerløw E, Bjørgaas MR, Nielsen EW, et al. Fear of hypoglycemia in women and men with type 1 diabetes. Nurs Res 2014:63:143-9.

26. Kahn MB, Cubbon RM, Mercer B, et al. Association of diabetes with increased all-cause mortality following primary percutaneous coronary intervention for ST-segment elevation myocardial infarction in the contemporary era. Diabetes Vasc Dis Res 2012;9:3-9.

27. Khunti K, Davies M, Majeed A, et al. Hypoglycemia and risk of cardiovascular disease and all-cause mortality in insulin-treated people with type 1 and type 2 diabetes: a cohort study. Diabetes Care 2015;38:316-22. 